\title{
Matrix inequalities for unitarily invariant norms
}

\section{Jianguo Zhao}

School of Mathematics and Statistics, Yangtze Normal University, Fulin, Chongqing 408100 China

e-mail: jgzhao_dj@163.com

Received 28 Apr 2018

Accepted 30 Aug 2018

ABSTRACT: In this study, we present some matrix inequalities for unitarily invariant norms. Firstly, we present an inequality for unitarily invariant norms. As a consequence of this result, Huang-Peng-Zou's result follows immediately. Furthermore, we also establish inequalities for weak log-majorizations and unitarily invariant norms related to question of Bourin's.

KEYWORDS: $t$-geometric mean, positive semidefinite matrices, weak log-majorization

MSC2010: 15A42 15A48 47A63

\section{INTRODUCTION}

Let $\mathscr{M}_{n}$ be the space of $n \times n$ complex matrices. Let $\lambda_{j}(A), j=1,2, \ldots, n$, be the eigenvalues of $A \in \mathscr{M}_{n}$ repeated according to multiplicity, and $|\lambda(A)|:=\left(\left|\lambda_{1}(A)\right|,\left|\lambda_{2}(A)\right|, \ldots,\left|\lambda_{n}(A)\right|\right)$ with $\left|\lambda_{1}(A)\right| \geqslant\left|\lambda_{2}(A)\right| \geqslant \cdots \geqslant\left|\lambda_{n}(A)\right|$. For $A \in \mathscr{M}_{n}$, the singular values of $A$ is denoted by $\sigma_{j}(A)$, $j=1,2, \ldots, n$, i.e., the eigenvalues of the positive semidefinite matrix $|A|=\left(A^{*} A\right)^{1 / 2}$, arranged in decreasing order and repeated according to multiplicity, where $A^{*}$ is the conjugate transpose of $A$. Let $\sigma(A):=\left(\sigma_{1}(A), \sigma_{2}(A), \ldots, \sigma_{n}(A)\right)$ be the vector of the singular values of $A$. For two Hermitian matrices $A, B \in \mathscr{M}_{n}, A \leqslant(<) B$ means $B-A$ is a positive semidefinite (definite) matrix. A norm $\|\cdot\|$ on $\mathscr{M}_{n}$ is called a unitarily invariant norm if $\|U A V\|=\|A\|$ for $A, U, V \in \mathscr{M}_{n}$ with $U, V$ are unitary matrices.

Let $\Phi(\cdot)$ be the corresponding symmetric gauge function of the unitarily invariant norms $\|\cdot\|$. Then $\|A\|=\Phi\left(\left\{\sigma_{i}(A)\right\}_{i=1}^{n}\right)$ for all $A \in \mathscr{M}_{n}$. Examples in this class are the Schatten $p$-norms and Ky Fan $k$-norms. $I_{n}$ is the identity matrix of $\mathscr{M}_{n}$. The usual operator norm denoted by $\|\cdot\|_{\infty}$ is $\|A\|_{\infty}=\sigma_{1}(A)$ for $A \in \mathscr{M}_{n}$.

Let us recall some definitions of majorization. Given a real vector $x=\left(x_{1}, x_{2}, \ldots, x_{n}\right) \in \mathbb{R}^{n}$, we rearrange its components as $x_{[1]} \geqslant x_{[2]} \geqslant \cdots \geqslant x_{[n]}$. For $x=\left(x_{1}, x_{2}, \ldots, x_{n}\right), y=\left(y_{1}, y_{2}, \ldots, y_{n}\right) \in \mathbb{R}^{n}$, if

$$
\sum_{i=1}^{k} x_{[i]} \leqslant \sum_{i=1}^{k} y_{[i]}, k=1,2, \ldots, n \text {, }
$$

then we say that $x$ is weakly majorized by $y$ and denotes by $x \prec_{\mathrm{w}} y$. If $x \prec_{\mathrm{w}} y$ and $\sum_{i=1}^{n} x_{i}=$ $\sum_{i=1}^{n} y_{i}$, then we say that $x$ is majorized by $y$ and denotes by $x \prec y$. Further, if $x=\left(x_{1}, x_{2}, \ldots, x_{n}\right)$, $y=\left(y_{1}, y_{2}, \ldots, y_{n}\right) \in \mathbb{R}_{+}^{n}$ and

$$
\prod_{i=1}^{k} x_{[i]} \leqslant \prod_{i=1}^{k} y_{[i]}, k=1,2, \ldots, n,
$$

then we say that $x$ is weakly log-majorized by $y$ and denotes by $x \prec_{w \log } y$. If $x \prec_{w \log } y$ and $\prod_{i=1}^{n} x_{i}=$ $\prod_{i=1}^{n} y_{i}$, then we say that $x$ is log-majorized by $y$ and denotes by $x \prec_{\log } y$. It is well-known that if $x \prec_{\mathrm{w} \log } y$, then $x \prec_{\mathrm{w}} y$.

Let $A \in \mathscr{M}_{n}$ be a Hermitian matrix with eigenvalues $\lambda_{j}(A)(j=1,2, \ldots, n)$. Then the spectral theorem states: there is a diagonal matrix $\Lambda=$ $\operatorname{diag}\left(\lambda_{1}(A), \lambda_{2}(A), \ldots, \lambda_{n}(A)\right)$ such that

$$
A=U \Lambda U^{*},
$$

where $U$ is a unitary matrix.

Let $f$ be a real continuous function on an interval $[a, b]$, if $A \in \mathscr{M}_{n}$ is a Hermitian matrix with eigenvalues $\lambda_{j}(A) \in[a, b], j=1,2, \ldots, n$. Then by the spectral theorem of $A, f(A)$ is defined by

$$
f(A)=U \Lambda_{f} U^{*},
$$

where $U$ is a unitary matrix and $\Lambda_{f}=$ $\operatorname{diag}\left(f\left(\lambda_{1}(A)\right), f\left(\lambda_{2}(A)\right), \ldots, f\left(\lambda_{n}(A)\right)\right)$.

Bhatia et al $^{1}$ proved that; if $A, B \in \mathscr{M}_{n}$ be two positive semidefinite matrices, then

$$
\left\|A^{m}+B^{m}\right\| \leqslant\left\|(A+B)^{m}\right\|
$$

holds for any positive integer $m$ and any unitarily invariant norm $\|\cdot\|$. Ando et $\mathrm{al}^{2}$ generalized inequality 
(1) that; if $A, B \in \mathscr{M}_{n}$ be two positive semidefinite matrices, then

$$
\left\|A^{p}+B^{p}\right\| \leqslant\left\|(A+B)^{p}\right\|
$$

holds for any positive real number $p$ with $1 \leqslant p<$ $\infty$ and any unitarily invariant norm $\|\cdot\|$. Bourin et $\mathrm{al}^{3}$ obtained a more generalization of inequality (2). They presented; if $A, B \in \mathscr{M}_{n}$ be two positive semidefinite matrices and $f:[0, \infty) \rightarrow[0, \infty)$ be a convex function with $f(0)=0$, then

$$
\|f(A)+f(B)\| \leqslant\|f(A+B)\|
$$

holds for any unitarily invariant norm $\|\cdot\|$. Recently, Huang et al ${ }^{4}$ obtained; if $A, B \in \mathscr{M}_{n}$ and suppose that $p, q$ be real numbers with $p>1$ and $1 / p+1 / q=$ 1 , then

$$
\begin{aligned}
& \left\|A|A|^{m-1}+B|B|^{m-1}\right\| \\
& \quad \leqslant\left\|\left(|A|^{m}+|B|^{m}\right)^{p / 2}\right\|^{1 / p} \cdot\left\|\left(\left|A^{*}\right|^{m}+\left|B^{*}\right|^{m}\right)^{q / 2}\right\|^{1 / q}
\end{aligned}
$$

holds for any positive integer $m$ and for any unitarily invariant norm $\|\cdot\|$. If $A=U|A|$ and $B=V|B|$ be the polar decompositions of $A$ and $B$, respectively. Then inequality (4) can be rewritten as

$$
\begin{aligned}
& \left\|U|A|^{m}+V|B|^{m}\right\| \\
& \quad \leqslant\left\|\left(|A|^{m}+|B|^{m}\right)^{p / 2}\right\|^{1 / p} \cdot\left\|\left(\left|A^{*}\right|^{m}+\left|B^{*}\right|^{m}\right)^{q / 2}\right\|^{1 / q} .
\end{aligned}
$$

On the other hand, Hayajneh et $\mathrm{al}^{5}$ and Liu et $\mathrm{al}^{6}$ were independently obtained: If $A_{i}, B_{i} \in \mathscr{M}_{n}$ be positive semidefinite matrices with $A_{i} B_{i}=B_{i} A_{i}, i=$ $1,2, \ldots, m$, then for all unitarily invariant norms $\|\cdot\|$,

$$
\begin{aligned}
& \left\|\left(\sum_{i=1}^{m} A_{i}^{1 / 2} B_{i}^{1 / 2}\right)^{2}\right\| \\
& \leqslant\left\|\left(\sum_{i=1}^{m} A_{i}\right)^{1 / 2}\left(\sum_{i=1}^{m} B_{i}\right)\left(\sum_{i=1}^{m} A_{i}\right)^{1 / 2}\right\| .
\end{aligned}
$$

Inequality (6) is a refinement of the following inequality obtained by Audenaert ${ }^{7}$ : If $A_{i}, B_{i} \in \mathscr{M}_{n}$ be positive semidefinite matrices with $A_{i} B_{i}=B_{i} A_{i}$, $i=1,2, \ldots, m$, then for all unitarily invariant norms $\|\cdot\|$,

$$
\left\|\left(\sum_{i=1}^{m} A_{i}^{1 / 2} B_{i}^{1 / 2}\right)^{2}\right\| \leqslant\left\|\left(\sum_{i=1}^{m} A_{i}\right)\left(\sum_{i=1}^{m} B_{i}\right)\right\| .
$$

$\mathrm{Hoa}^{8}$ and Lin $^{9}$ presented different proofs for inequality (7), respectively. Inequality (7) gave an affirmative answer to Bourin's question. Given two positive semidefinite matrices $A, B \in \mathscr{M}_{n}$ and two positive real numbers $p, q$, is it true that

$$
\left\|A^{p+q}+B^{p+q}\right\| \leqslant\left\|\left(A^{p}+B^{p}\right)\left(A^{q}+B^{q}\right)\right\| ?
$$

Refinements and improvements for unitarily invariant norms have been extensively studied. Many researchers, e.g., Bhatia ${ }^{10}$, Fujii ${ }^{11}, \mathrm{Hu}^{12,13}$, Kapil ${ }^{14}$, Kittaneh $^{15}$, Kuzma ${ }^{16}$, Matharu ${ }^{17}$, paid attention to the improvement and generalization of inequalities for unitarily invariant norms.

In this study, we present some matrix inequalities for unitarily invariant norms. We present a generalization of inequality (5) and establish inequalities for weak log-majorizations and unitarily invariant norms related to Bourin's question.

\section{MATRIX INEQUALITIES FOR UNITARILY INVARIANT NORMS}

This section mainly presents a generalization of inequality (5) for unitarily invariant norms and inequalities for weak log-majorizations and unitarily invariant norms related to Bourin's question. To achieve the goal, we require lemmas presented in Ref. 18.

Lemma 1 If $A, B \in \mathscr{M}_{n}$ with $A, B \geqslant 0$, then the matrix $\left[\begin{array}{cc}A & X \\ X^{*} & B\end{array}\right]$ is positive semidefinite if and only if $X=$ $A^{1 / 2} K B^{1 / 2}$ for some contraction $K$, i.e., $K^{*} K \leqslant I_{n}$.

Lemma $2 A \geqslant 0$ if and only if $\left[\begin{array}{ll}A & A \\ A & A\end{array}\right] \geqslant 0$.

The next lemma was obtained by Horn ${ }^{19}$.

Lemma 3 If $A, B \in \mathscr{M}_{n}$, then

$$
\sigma(A B) \prec_{\log }\left\{\sigma_{i}(A) \sigma_{i}(B)\right\}_{i=1}^{n} .
$$

The Lemma 4 was obtained by Matharu et al ${ }^{17}$.

Lemma 4 If $A, B \in \mathscr{M}_{n}$ with $A, B>0$ and $t \in[0,1]$, then

$$
\lambda\left(A \sharp_{t} B\right) \prec_{w \log } \lambda\left(A^{1-t} B^{t}\right),
$$

where $A \sharp_{t} B=A^{1 / 2}\left(A^{-1 / 2} B A^{-1 / 2}\right)^{t} A^{1 / 2}$ is the $t$ geometric mean of $A$ and $B{ }^{20}$.

The next lemma was given by $\mathrm{Hiai}^{21}$.

Lemma 5 If $A, B \in \mathscr{M}_{n}$ with $A, B>0$ and $t \in[0,1]$, then

$$
\lambda\left(\left(A^{1 / 2} B A^{1 / 2}\right)^{r}\right) \prec_{w \log } \lambda\left(A^{r / 2} B^{r} A^{r / 2}\right),
$$

for $r \geqslant 1$. 
The next Lemma 6 is the famous Weyl's theorem ${ }^{19}$ on the singular values and the eigenvalues of a matrix.

Lemma 6 If $A \in \mathscr{M}_{n}$, then

$$
|\lambda(A)| \prec_{\log } \sigma(A) .
$$

In the following, we present the famous Fan dominance theorem ${ }^{19}$.

Lemma 7 If $A, B \in \mathscr{M}_{n}$, then

$$
\sigma(A) \prec_{\mathrm{w}} \sigma(B) \Longleftrightarrow\|A\| \leqslant\|B\|
$$

for any unitarily invariant norm $\|\cdot\|$.

It is now time to present the following theorem.

Theorem 1 If $A, B \in \mathscr{M}_{n}$ and $f:[0, \infty) \rightarrow[0, \infty)$ is a continuous function with $f(0)=0$, then

$$
\begin{gathered}
\|U f(|A|)+V f(|B|)\| \leqslant\left\|(f(|A|)+f(|B|))^{p / 2}\right\|^{1 / p} \\
\cdot\left\|\left(f\left(\left|A^{*}\right|\right)+f\left(\left|B^{*}\right|\right)\right)^{q / 2}\right\|^{1 / q}
\end{gathered}
$$

holds for positive real numbers $p$, $q$ with $1 / p+1 / q=1$ and any unitarily invariant norm $\|\cdot\|$, where $U$ and $V$ are unitary matrices with $A=U|A|$ and $B=V|B|$, respectively.

Proof: Let $A=U|A|$ and $B=V|B|$ be the polar decompositions of $A$ and $B$, respectively. By Lemma 2 , we have

$$
\left[\begin{array}{cc}
|A| & A^{*} \\
A & \left|A^{*}\right|
\end{array}\right]=\left[\begin{array}{cc}
I_{n} & 0 \\
0 & U
\end{array}\right]\left[\begin{array}{cc}
A & |A| \\
|A| & |A|
\end{array}\right]\left[\begin{array}{cc}
I_{n} & 0 \\
0 & U^{*}
\end{array}\right] \geqslant 0 .
$$

Putting

$$
W=\frac{1}{\sqrt{2}}\left[\begin{array}{cc}
I_{n} & -I_{n} \\
I_{n} & I_{n}
\end{array}\right],
$$

then $W$ is a unitary matrix and

$$
W\left[\begin{array}{cc}
|A| & 0 \\
0 & 0
\end{array}\right] W^{*}=\frac{1}{2}\left[\begin{array}{ll}
|A| & |A| \\
|A| & |A|
\end{array}\right] .
$$

Combining equalities (10) with (11), we obtain

$$
\frac{1}{2}\left[\begin{array}{cc}
|A| & A^{*} \\
A & \left|A^{*}\right|
\end{array}\right]=\left[\begin{array}{cc}
I_{n} & 0 \\
0 & U
\end{array}\right] W\left[\begin{array}{cc}
|A| & 0 \\
0 & 0
\end{array}\right] W^{*}\left[\begin{array}{cc}
I_{n} & 0 \\
0 & U^{*}
\end{array}\right] .
$$

Since $f$ is a nonnegative function on $[0, \infty)$ with $f(0)=0$, by equality (12) we obtain

$$
f\left(\frac{1}{2}\left[\begin{array}{cc}
|A| & A^{*} \\
A & \left|A^{*}\right|
\end{array}\right]\right)=\frac{1}{2}\left[\begin{array}{cc}
f(|A|) & f(|A|) U^{*} \\
U f(|A|) & f\left(\left|A^{*}\right|\right)
\end{array}\right] .
$$

Similarly, we also have

$$
f\left(\frac{1}{2}\left[\begin{array}{cc}
|B| & B^{*} \\
B & \left|B^{*}\right|
\end{array}\right]\right)=\frac{1}{2}\left[\begin{array}{cc}
f(|B|) & f(|B|) V^{*} \\
V f(|B|) & f\left(\left|B^{*}\right|\right)
\end{array}\right] .
$$

It follows from equalities (13) and (14) that

$$
\begin{aligned}
& {\left[\begin{array}{cc}
f(|A|)+f(|B|) & f(|A|) U^{*}+f(|B|) V^{*} \\
U f(|A|)+V f(|B|) & f\left(\left|A^{*}\right|\right)+f\left(\left|B^{*}\right|\right)
\end{array}\right]} \\
& \quad=2 f\left(\frac{1}{2}\left[\begin{array}{cc}
|A| & A^{*} \\
A & \left|A^{*}\right|
\end{array}\right]\right)+2 f\left(\frac{1}{2}\left[\begin{array}{cc}
|B| & B^{*} \\
B & \left|B^{*}\right|
\end{array}\right]\right) \geqslant 0 .
\end{aligned}
$$

By inequality (15) and Lemma 1, there is a contraction $K$ such that

$$
\begin{aligned}
& f(|A|) U^{*}+f(|B|) V^{*}= \\
& \quad(f(|A|)+f(|B|))^{1 / 2} K\left(f\left(\left|A^{*}\right|\right)+f\left(\left|B^{*}\right|\right)\right)^{1 / 2},
\end{aligned}
$$

or equivalently,

$$
\begin{aligned}
& U f(|A|)+V f(|B|)= \\
& \left(f\left(\left|A^{*}\right|\right)+f\left(\left|B^{*}\right|\right)\right)^{1 / 2} K^{*}(f(|A|)+f(|B|))^{1 / 2} .
\end{aligned}
$$

According to (16), we have

$$
\begin{aligned}
& \prod_{j=1}^{k} \sigma_{j}(U f(|A|)+V f(|B|)) \\
& \leqslant \prod_{j=1}^{k} \sigma_{j}\left(\left(f\left(\left|A^{*}\right|\right)+f\left(\left|B^{*}\right|\right)\right)^{\frac{1}{2}}\right) \sigma_{j}\left(K^{*}(f(|A|)+f(|B|))^{\frac{1}{2}}\right) \\
& \leqslant \prod_{j=1}^{k} \sigma_{j}\left(\left(f\left(\left|A^{*}\right|\right)+f\left(\left|B^{*}\right|\right)\right)^{\frac{1}{2}}\right) \sigma_{j}\left((f(|A|)+f(|B|))^{\frac{1}{2}}\right),
\end{aligned}
$$

for $k=1,2, \ldots, n$, the first inequality is due to Lemma 3, and the second by the contractive of $K^{*}$.

Since weak log-majorization implies weak majorization, we obtain the following weak majorization from (17).

$$
\begin{aligned}
& \sigma(U f(|A|)+V f(|B|)) \prec_{\mathrm{w}} \\
& \quad\left\{\sigma_{j}\left(\left(f\left(\left|A^{*}\right|\right)+f\left(\left|B^{*}\right|\right)\right)^{\frac{1}{2}}\right) \sigma_{j}\left((f(|A|)+f(|B|))^{\frac{1}{2}}\right)\right\}_{j=1}^{n} .
\end{aligned}
$$

Let $\Phi(\cdot)$ be the corresponding symmetric gauge function for the unitarily invariant norm $\|\cdot\|$. By the Cauchy-Schwarz inequality for $\Phi(\cdot)$ we have ${ }^{22}$

$$
\begin{aligned}
& \Phi\left(\left\{\sigma_{j}\left(\left(f\left(\left|A^{*}\right|\right)+f\left(\left|B^{*}\right|\right)\right)^{\frac{1}{2}}\right) \sigma_{j}\left((f(|A|)+f(|B|))^{\frac{1}{2}}\right)\right\}_{j=1}^{n}\right) \\
& \leqslant \Phi\left(\sigma\left(\left(f\left(\left|A^{*}\right|\right)+f\left(\left|B^{*}\right|\right)\right)^{\frac{p}{2}}\right)\right)^{\frac{1}{p}} \Phi\left(\sigma\left((f(|A|)+f(|B|))^{\frac{q}{2}}\right)\right)^{\frac{1}{q}}
\end{aligned}
$$


for positive real numbers $p, q$ with $1 / p+1 / q=1$. On the other hand, by inequality (18), we obtain

$\Phi(\sigma(U f(|A|)+V f(|B|))) \leqslant$

$\Phi\left(\left\{\sigma_{j}\left(\left(f\left(\left|A^{*}\right|\right)+f\left(\left|B^{*}\right|\right)\right)^{\frac{1}{2}}\right) \sigma_{j}\left((f(|A|)+f(|B|))^{\frac{1}{2}}\right)\right\}_{j=1}^{n}\right)$.

Noting that $\Phi(\sigma(A))=\|A\|$ for $A \in \mathscr{M}_{n}$ and (19) and (20) imply the desired result (9).

$$
\begin{aligned}
& \|U f(|A|)+V f(|B|)\| \leqslant \\
& \quad\left\|(f(|A|)+f(|B|))^{\frac{p}{2}}\right\|^{\frac{1}{p}}\left\|\left(f\left(\left|A^{*}\right|\right)+f\left(\left|B^{*}\right|\right)\right)^{\frac{q}{2}}\right\|^{\frac{1}{q}} .
\end{aligned}
$$

Remark 1 Putting $f(x)=x^{m}$ for some positive integer number $m$, (5) follows immediately from (10).

Remark 2 Let $f$ be a nonnegative convex function on $[0, \infty)$ with $f(0)=0$ and $p=q=2$. Then by inequality (10) we have

$$
\begin{aligned}
& \|U f(|A|)+V f(|B|)\| \\
& \quad \leqslant\|f(|A|)+f(|B|)\|^{\frac{1}{2}}\left\|f\left(\left|A^{*}\right|\right)+f\left(\left|B^{*}\right|\right)\right\|^{\frac{1}{2}},
\end{aligned}
$$

and by inequalities (3) and (21), the following inequality holds

$$
\begin{aligned}
\| U f(|A|) & +V f(|B|) \| \\
& \leqslant\|f(|A|+|B|)\|^{\frac{1}{2}}\left\|f\left(\left|A^{*}\right|+\left|B^{*}\right|\right)\right\|^{\frac{1}{2}} .
\end{aligned}
$$

If $A, B \geqslant 0$, then $U=V=I_{n}$. Hence, inequality (3) follows immediately from (22).

Next, we give weak log-majorization inequalities for positive semidefinite matrices.

Theorem 2 If $A, B \in \mathscr{M}_{n}$ with $A, B>0$ and $t \in[0,1]$, then

$$
\begin{aligned}
\lambda\left(\left(A_{t} B\right)^{r}\right) \prec_{w \log } \lambda & \left(\left(B^{r t s / 2} A^{(1-t) r s} B^{r t s / 2}\right)^{1 / s}\right) \\
& \prec_{w \log } \lambda\left(\left|A^{(1-t) r s} B^{r t s}\right|^{1 / s}\right),
\end{aligned}
$$

holds for positive $r$, $s$ with $r s \geqslant 1$.

Proof: By Lemma 4 we have, for $k=1,2, \ldots, n$,

$$
\begin{aligned}
\prod_{j=1}^{k} \lambda_{j}\left(A \sharp_{t} B\right) \leqslant \prod_{j=1}^{k} & \lambda_{j}\left(A^{1-t} B^{t}\right) \\
& =\prod_{j=1}^{k} \lambda_{j}\left(B^{t} A^{1-t}\right) \\
& =\prod_{j=1}^{k} \lambda_{j}\left(B^{t / 2} A^{1-t} B^{t / 2}\right) .
\end{aligned}
$$

By Lemma 5 we obtain, for $r s \geqslant 1$,

$$
\begin{gathered}
\prod_{j=1}^{k} \lambda_{j}^{r s}\left(B^{t / 2} A^{1-t} B^{t / 2}\right)=\prod_{j=1}^{k} \lambda_{j}\left(\left(B^{t / 2} A^{1-t} B^{t / 2}\right)^{r s}\right) \\
\leqslant \prod_{j=1}^{k} \lambda_{j}\left(B^{r s t / 2} A^{(1-t) r s} B^{r s t / 2}\right) .
\end{gathered}
$$

Combining inequalities (24) and (25), we obtain for $k=1,2, \ldots, n$ and $r s \geqslant 1$,

$$
\prod_{j=1}^{k} \lambda_{j}^{r}\left(A_{\sharp_{t}} B\right) \leqslant \prod_{j=1}^{k} \lambda_{j}\left(\left(B^{r s t / 2} A^{(1-t) r s} B^{r s t / 2}\right)^{1 / s}\right) .
$$

On the other hand, by Lemma 6, we have

$$
\begin{aligned}
\prod_{j=1}^{k} \lambda_{j}\left(B^{r s t / 2} A^{(1-t) r s} B^{r s t / 2}\right)= & \prod_{j=1}^{k} \lambda_{j}\left(A^{(1-t) r s} B^{r t s}\right) \\
& \leqslant \prod_{j=1}^{k} \sigma_{j}\left(A^{(1-t) r s} B^{r s t}\right),
\end{aligned}
$$

or equivalently,

$$
\begin{gathered}
\prod_{j=1}^{k} \lambda_{j}\left(\left(B^{\frac{r s t}{2}} A^{(1-t) r s} B^{\frac{r s t}{2}}\right)^{\frac{1}{s}}\right)=\prod_{j=1}^{k} \lambda_{j}^{\frac{1}{s}}\left(A^{(1-t) r s} B^{r s t}\right) \\
\leqslant \prod_{j=1}^{k} \sigma_{j}^{\frac{1}{s}}\left(A^{(1-t) r s} B^{r s t}\right) \\
=\prod_{j=1}^{k} \lambda_{j}\left(\left|A^{(1-t) r s} B^{r s t}\right|^{\frac{1}{s}}\right) .
\end{gathered}
$$

Thus the desired inequality (23) follows from (26) and (27).

Since weak log-majorization implies weak majorization, Theorem 2 and Lemma 7 imply the following theorem:

Theorem 3 If $A, B \in \mathscr{M}_{n}$ with $A, B>0$ and $t \in[0,1]$, then for all unitarily invariant norms $\|\cdot\|$ on $\mathscr{M}_{n}$,

$$
\begin{aligned}
\left\|\left(A \sharp_{t} B\right)^{r}\right\| \leqslant\left\|\left(B^{r s t / 2} A^{(1-t) r s} B^{r s t}\right)^{1 / s}\right\| \\
\leqslant\left\|\left.|| A^{(1-t) r s} B^{r s t}\right|^{1 / s}\right\|
\end{aligned}
$$

holds for positive $r, s$ with $r s \geqslant 1$.

Remark 3 Hoa obtained the following result ${ }^{8}$; if $A, B \in \mathscr{M}_{n}$ with $A, B>0$ and $t \in[0,1]$, then for all 
unitarily invariant norms $\|\cdot\|$ on $\mathscr{M}_{n}$,

$$
\begin{aligned}
\left\|\left(A \sharp_{t} B\right)^{r}\right\| & \leqslant\left\|\left(B^{r s t / 2} A^{(1-t) r s} B^{r s t}\right)^{1 / s}\right\| \\
& \leqslant\left\|\left.A^{(1-t) r s} B^{r s t}\right|^{1 / s}\right\|
\end{aligned}
$$

holds for $r \geqslant 1, s>0$. Since $\{(s, r) \mid 0<r<1<$ $r s\} \cap\{(s, r) \mid r \geqslant 1, s>0\}=\varnothing,(28)$ is a complement of (29).

Theorem 4 Let $A_{i}, B_{i} \in \mathscr{M}_{n}$ be positive definite matrices, $i=1,2, \ldots, m$. Then

$$
\begin{gathered}
\lambda\left(\left(\sum_{i=1}^{m} A_{i} \sharp_{t} B_{i}\right)^{r}\right) \\
\prec_{w \log } \lambda\left(\left(\left(\sum_{i=1}^{m} B_{i}\right)^{\frac{r s t}{2}}\left(\sum_{i=1}^{m} A_{i}\right)^{r s t}\left(\sum_{i=1}^{m} B_{i}\right)^{\frac{r s t}{2}}\right)^{\frac{1}{s}}\right) \\
\prec_{w \log } \lambda\left(\left|\left(\sum_{i=1}^{m} A_{i}\right)^{(1-t) r s}\left(\sum_{i=1}^{m} B_{i}\right)^{r s t}\right|^{\frac{1}{s}}\right),
\end{gathered}
$$

where for positive $r, s$ with $r s \geqslant 1$ and $t \in[0,1]$.

Proof: By the monotonicity of the operator mean $\sharp_{t}$ for $t \in[0,1]$, we have

$$
\sum_{i=1}^{m} A_{i} \sharp_{t} B_{i} \leqslant\left(\sum_{i=1}^{m} A_{i}\right) \sharp_{t}\left(\sum_{i=1}^{m} B_{i}\right),
$$

which implies

$$
\lambda_{j}\left(\sum_{i=1}^{m} A_{i} \sharp_{t} B_{i}\right) \leqslant \lambda_{j}\left(\left(\sum_{i=1}^{m} A_{i}\right) \sharp_{t}\left(\sum_{i=1}^{m} B_{i}\right)\right)
$$

for $j=1,2, \ldots, n$. According to Theorem 2, we obtain

$$
\begin{gathered}
\lambda\left(\left(\left(\sum_{i=1}^{m} A_{i}\right) \sharp_{t}\left(\sum_{i=1}^{m} B_{i}\right)\right)^{r}\right) \\
\prec_{w \log } \lambda\left(\left(\left(\sum_{i=1}^{m} B_{i}\right)^{\frac{r s t}{2}}\left(\sum_{i=1}^{m} A_{i}\right)^{r s t}\left(\sum_{i=1}^{m} B_{i}\right)^{\frac{r s t}{2}}\right)^{\frac{1}{s}}\right) \\
\prec_{w \log } \lambda\left(\left|\left(\sum_{i=1}^{m} A_{i}\right)^{(1-t) r s}\left(\sum_{i=1}^{m} B_{i}\right)^{r s t}\right|^{\frac{1}{s}}\right) .
\end{gathered}
$$

Combining inequalities (31) and (32), we obtain the desired inequality (30).

Since weak log-majorization implies weak majorization, by Theorem 4 and Lemma 7, we obtain the following theorem.
Theorem 5 If $A_{i}, B_{i} \in \mathscr{M}_{n}$ with $A_{i}, B_{i}>0, \quad i=$ $1,2, \ldots, m$, then

$$
\begin{aligned}
\left\|\left(\sum_{i=1}^{m} A_{i} \sharp_{t} B_{i}\right)^{r}\right\| & \\
\leqslant \|\left(\left(\sum_{i=1}^{m} B_{i}\right)^{\frac{r s t}{2}}\right. & \left.\left(\sum_{i=1}^{m} A_{i}\right)^{(1-t) r s}\left(\sum_{i=1}^{m} B_{i}\right)^{\frac{r s t}{2}}\right)^{\frac{1}{s}} \| \\
\leqslant & \left\|\left.\left(\sum_{i=1}^{m} A_{i}\right)^{(1-t) r s}\left(\sum_{i=1}^{m} B_{i}\right)^{r s t}\right|^{\frac{1}{s}}\right\|
\end{aligned}
$$

holds for any unitarily invariant norm $\|\cdot\|$, positive $r$, $s$ with $r s \geqslant 1$, and $t \in[0,1]$.

Taking $r=2$ and $s=1$ in Theorem 5 , we have the following corollary.

Corollary 1 If $A_{i}, B_{i} \in \mathscr{M}_{n}$ with $A_{i}, B_{i}>0, i=$ $1,2, \ldots, m$, then

$$
\begin{array}{r}
\left\|\left(\sum_{i=1}^{m} A_{i} \sharp_{t} B_{i}\right)^{2}\right\| \\
\leqslant\left\|\left(\sum_{i=1}^{m} B_{i}\right)^{t}\left(\sum_{i=1}^{m} A_{i}\right)^{2(1-t)}\left(\sum_{i=1}^{m} B_{i}\right)^{t}\right\| \\
\leqslant\left\|\left(\sum_{i=1}^{m} A_{i}\right)^{2(1-t)}\left(\sum_{i=1}^{m} B_{i}\right)^{2 t}\right\|
\end{array}
$$

holds for any unitarily invariant norm and $t \in[0,1]$.

Since $A \sharp_{t} B=A^{1-t} B^{t}$ when $A B=B A$ for $A, B>0$, the following remark holds.

Remark 4 Let $A_{i}, B_{i} \in \mathscr{M}_{n}$ be positive definite matrices with $A_{i} B_{i}=B_{i} A_{i}, i=1,2, \ldots, m$. From (3), by taking $f(x)=x^{2}$, we have

$$
\left\|\sum_{i=1}^{m}\left(A_{i}^{1-t} B_{i}^{t}\right)^{2}\right\| \leqslant\left\|\left(\sum_{i=1}^{m} A_{i}^{1-t} B_{i}^{t}\right)^{2}\right\| .
$$

Combining inequalities (33) and (34), we obtain

$$
\begin{array}{r}
\left\|\sum_{i=1}^{m}\left(A_{i}^{1-t} B_{i}^{t}\right)^{2}\right\| \\
\leqslant\left\|\left(\sum_{i=1}^{m} B_{i}\right)^{t}\left(\sum_{i=1}^{m} A_{i}\right)^{2(1-t)}\left(\sum_{i=1}^{m} B_{i}\right)^{t}\right\| \\
\leqslant\left\|\left(\sum_{i=1}^{m} A_{i}\right)^{2(1-t)}\left(\sum_{i=1}^{m} B_{i}\right)^{2 t}\right\| .
\end{array}
$$


Inequality (35) is a generalization of inequalities (6) and (7). On the other hand, let $A$ and $B$ be two positive definite matrices and $p, q$ be two positive real numbers. Taking $m=2, t=\frac{1}{2}, A_{1}=A^{p}, B_{1}=A^{q}$, $A_{2}=B^{p}, B_{2}=B^{q}$ in inequality (35), we have

$$
\begin{array}{r}
\left\|A^{p+q}+B^{p+q}\right\| \leqslant\left\|\left(A^{p}+B^{p}\right)^{\frac{1}{2}}\left(A^{q}+B^{q}\right)\left(A^{p}+B^{p}\right)^{\frac{1}{2}}\right\| \\
\leqslant\left\|\left(A^{p}+B^{p}\right)\left(A^{q}+B^{q}\right)\right\|,
\end{array}
$$

which is (8). This gives an affirmative answer to Bourin's question.

Acknowledgements: The author would like to thank the editors and referees for their comments and suggestions which improve this manuscript largely. This work is supported by the National Natural Foundation of China (Grant No. 11161040).

\section{REFERENCES}

1. Bhatia R, Kittaneh F (1998) Norm inequalities for positive operators. Lett Math Phys 43, 225-31.

2. Ando T, Zhan X (1999) Norm inequalities related to operator monotone functions. Math Ann 315, 771-80.

3. Bourin JC, Uchiyama M (2007) A matrix subadditivity inequality for $f(A+B)$ and $f(A)+f(B)$. Linear Algebra Appl 423, 512-8.

4. Huang J, Peng Y, Zou L (2014) A note on norm inequalities for positive operators. J Inequal Appl 171, 1-4.

5. Hayajneh M, Hayajneh S, Kittaneh F (2017) Remarks on some norm inequalities for positive semidefinite matrices and questions of Bourin. Math Inequal Appl 20, 225-32.

6. Liu J, Wang Q, Sun F (2017) On Hayajneh and Kittaneh's conjecture on unitarily invariant norm. $J$ Math Inequal 11, 1019-22.

7. Audenaert K (2015) A norm inequality for pairs of commuting positive semidefinite matrices. Electron $J$ Linear Al 30, 80-4.

8. Hoa D (2016) An inequality for t-geometric means. Math Inequal Appl 19, 765-8.

9. Lin M (2016) Remarks on two recent results of Audenaert. Linear Algebra Appl 489, 24-9.

10. Bhatia R, Lim Y, Yamazaki T (2016) Some norm inequalities for matrix means. Linear Algebra Appl 501, 112-22.

11. Fujii JI, Seo Y, Yamazaki T (2016) Norm inequalities for matrix geometrc means of positive definite matrices. Linear Multilinear A 64, 512-26.

12. $\mathrm{Hu} X$ (2012) Some inequalities for unitarily invariant norms. $J$ Math Inequal 6, 615-23.

13. Hu X, Xue J (2015) A note on reverses of Young type inequalities. J Inequal Appl 98, 1-6.
14. Kapil Y, Conde C, Moslehian MS, Singh M, Sababheh M (2017) Norm inequalities related to Heron and Heinz means. Mediterr J Math 213, 1-18.

15. Kittaneh F, Moslehian MS, Sababheh M (2017) Unitarily invariant norm inequalities for elementary operators involving $G_{1}$ operators. Linear Algebra Appl 513, 84-95.

16. Kuzma B, Petek T (2017) Maps preserving unitarily invariant norms of Jordan products of matrices. $J$ Math Anal Appl 455, 1579-96.

17. Matharu J, Aujla J (2012) Some inequalities for unitarily invariant norm. Linear Algebra Appl 436, 1623-31.

18. Bhatia R (2007) Positive Definite Matrices, Princeton University Press.

19. Zhan X (2008) Matrix Theory, Higher Education Press. [in Chinese]

20. Kubo F, Ando T (1980) Means of positive linear operators. Math Ann 246, 205-24.

21. Hiai F (1997) Log-majorizations and norm inequalities for exponential operators. In: Linear Operators, Banach Center Publications 38, Polish Academy of Sciences, pp 119-81.

22. Bhatia R (1997) Matrix Analysis, Springer-Verlag. 